\title{
Double Exponential Formulas for Numerical Integration
}

\author{
By
}

Hidetosi TAKAHASI* and Masatake MoRI

\begin{abstract}
A family of numerical quadrature formulas is introduced by application of the trapezoidal rule to infinite integrals which result from the given integrals $\int_{a}^{b} f(x) d x$ by suitable variable transformations $x=\phi(u)$. These formulas are characterized by having double exponential asymptotic behavior of the integrands in the resulting infinite integrals as $u \rightarrow \pm \infty$, and it is shown both analytically and numerically that such formulas are generally optimal with respect to the ecomony of the number of sampling points.
\end{abstract}

\section{§1. Introduction}

For the numerical evaluation of an integral over an infinite interval $(-\infty, \infty)$

$$
I=\int_{-\infty}^{\infty} g(u) d u
$$

in which $g(u)$ is analytic over $(-\infty, \infty)$, it is known [1] that the uniformly divided trapezoidal formula

$$
I_{h}=h \sum_{n=-\infty}^{\infty} g(n h)
$$

is optimal in efficiency among quadrature formulas having the same density of sampling points, and hence application of (1.2) after carrying out suitable transformation on the variable $x$ gives a very efficient quadrature formula for finite or infinite interval. Such quadrature formulas are often

Received September 17, 1973.

* Faculty of Science, University of Tokyo, Tokyo. 
superior to the Gaussian and other formulas based on polynomial fitting of the integrand in that they are rather insensitive to the singularities which may be present in the original integrand at one or both ends of the interval as was noted by C. Schwartz [2]. In a preceding paper [3], we have given a general recipe for application of variable transform to the numerical integration based on the above ideas together with an error analysis for these formulas.

Obviously the efficiency of such quadrature formulas, i.e. the number of sampling points required for obtaining a given accuracy, strongly depends on the mapping function $\phi(u)$ being used, and there seems to have been no systematic theory which can tell which mapping function to use for a given integrand. The purpose of the present paper is to show, on the basis of an error analysis using contour integral method, that the mapping is in a certain sense optimal when the transformed integrand function behaves in a double exponential way in the infinite sum, and to give a number of practical mapping functions that give such good quadrature formulas.

Let the given integral be

$$
I=\int_{a}^{b} f(x) d x
$$

and let a variable transform

$$
x=\phi(u) \text { where } \phi(-\infty)=a, \phi(\infty)=b
$$

be applied to (1.3), so as to change the interval $(a, b)$ into the infinite interval $(-\infty, \infty)$ so that

$$
I=\int_{-\infty}^{\infty} g(u) d u
$$

where

$$
g(u)=f(\phi(u)) \phi^{\prime}(u) .
$$

One or both of the limits $a$ and $b$ in the original integral may be infinite. Application of the trapezoidal rule to (1.5) with mesh size $h$ yields a quadrature formula

$$
I_{h}=h \sum_{n=-\infty}^{\infty} f(\phi(n h)) \phi^{\prime}(n h) .
$$


Obviously, the infinite sum must be appropriately truncated in actual application.

The degree of approximation of the formula (1.7) to true value (1.3) can be evaluated $[3]$ in a form of a contour integral

$$
\Delta I_{h}=\frac{1}{2 \pi i} \int_{\hat{C}} \hat{\Phi}(w) f(\phi(w)) \phi^{\prime}(w) d w
$$

where

$$
\hat{\Phi}(w)=\left\{\begin{array}{l}
\frac{-2 \pi i}{1-\exp (-2 \pi i w / h)} \simeq+2 \pi i \exp (+2 \pi i w / h) ; \operatorname{Im} w>0 \\
\frac{+2 \pi i}{1-\exp (+2 \pi i w / h)} \simeq-2 \pi i \exp (-2 \pi i w / h) ; \operatorname{Im} w<0 .
\end{array}\right.
$$

The path $\hat{C}$ of integration should consists of any two infinite paths running in both sides of the real axis bounding an infinitely extended strip such that $f(\phi(w)) \phi^{\prime}(w)$ is regular over the strip (Fig. 1). Now, if we know

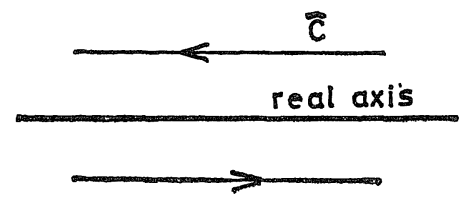

Fig. 1 Path $\hat{C}$ of the integral (1.8)

the singular points $w_{0}$ of $g(w)$ which lies nearest to the real axis in the $w$-plane, the contour integral (1.8), which gives the quadrature error $\Delta I_{h}$, can be roughly estimated according to a formula

$$
\left|\Delta I_{h}\right| \simeq \exp \left(-\frac{2 \pi}{h}\left|\operatorname{Im} w_{0}\right|\right)
$$

by making the path $\hat{C}$ pass through the saddle point that is located in the close neighborhood of $w_{0}$. The saddle point appears owing to the rapid decay of $|\hat{\Phi}(w)| \simeq 2 \pi \exp (-2 \pi|\operatorname{Im} w| / h)$ as $w$ goes away from the real axis and the steep growth of $g(w)$ towards the singularity at $w_{0}[1]$. If specifically $w_{0}$ is a simple pole of $g(w)$ having a residue $A$, we have $\left|\Delta I_{h}\right|$ $\simeq|A| \exp \left(-2 \pi\left|\operatorname{Im} w_{0}\right| / h\right)$. In any case the problem to estimate the error 
is reduced to that of finding the position $w_{0}$ of the singular point of $g(w)$ which is located nearest to the real axis in the $w$-plane.

In evaluating the various quadrature formulas obtained from various types of mapping functions $\phi(u)$, two generally conflicting factors have always to be taken into account. As have been already shown [3], the practical quadrature error consists of two terms, namely (1) the proper error $\Delta I_{h}$ of the original quadrature formula involving infinite summation, and (2) the error $\Delta I_{t}$ caused by the truncation of the infinite sum. In order to keep the truncation error sufficiently small and still not to have to use too many terms in the sum, one may wish to have the summand in (1.7) to die out very rapidly as $n$ goes to infinity, and this requirement favors any mapping function $\phi(u)$ which tends to the limiting values as fast as possible. The extreme in this direction is obviously the use of a mapping function which maps the original interval onto a finite interval as has been proposed by Iri, Moriguti and Takasawa [4]. In this case no truncation error exists since the quadrature formula consists of only a finite sum. In order to keep the quadrature error of the ideal formula involving infinite sum, on the other hand, it will be shown that it is usually better to use formulas based on mapping functions which tend to the limiting values more or less slowly. For the type of mapping functions $x=1 /\{1+$ $\left.\exp \left(-c u^{2 n+1}\right)\right\}$, for example, the asymptotic behavior of $\Delta I_{h}$ for $h \rightarrow 0$ will be shown to have a form

$$
\left|\Delta I_{h}\right| \propto \exp (-a / h) .
$$

The formula which involves a finite sum has an error formula with asymptotic form $[4]$

$$
\left|\Delta I_{h}\right| \propto \exp (-a / \sqrt{h})
$$

\section{§2. The Best Formula for Integrals over the Finite Interval $(-1,1)$}

Before going into a general discussion of the best mapping function, we will compare several different mapping functions for the integral

$$
I=\int_{-1}^{1} f(x) d x \quad(f(x): \text { analytic on }(-1,1))
$$


to serve heuristic purposes. $f(x)$ may have algebraic or logarithmic singularities at $x= \pm 1$ as long as it is integrable over $(-1,1)$.

$$
\begin{aligned}
& x=\tanh u^{m}, m=1,3,5, \ldots \\
& \quad \text { for which }|g(u)| \simeq \exp \left(-|u|^{m}\right) \text { as } u \rightarrow \pm \infty
\end{aligned}
$$

By this mapping function we have

$$
I=\int_{-\infty}^{\infty} f\left(\tanh u^{m}\right) \frac{m u^{m-1}}{\cosh ^{2} u^{m}} d u \text {. }
$$

If the trapezoidal rule with a step size $h$ is applied to (2.a.2) we have

$$
I_{h}=h \sum_{n=-\infty}^{\infty} f\left(\tanh n^{m} h^{m}\right) \frac{m n^{m-1} h^{m-1}}{\cosh ^{2}\left(n^{m} h^{m}\right)}
$$

The function for $m=1$

$$
z=\tanh w
$$

maps the whole $z$-plane onto an infinite number of strips $W_{k}$ parallel to the real axis:

$$
W_{k}=\left\{w \mid\left(k-\frac{1}{2}\right) \pi<\operatorname{Im} w<\left(k+\frac{1}{2}\right) \pi\right\} ; \quad k=0, \pm 1, \pm 2, \ldots
$$

In Fig. 2 we show the strip $W_{0}$ with the images of the lines parallel to the real and the imaginary axes in the $z$-plane. Take $m=3$ for $m>1$, for example. The mapping by $z=\tanh w^{3}$ is obtainable by composing the two mappings $z=\tanh \zeta, \zeta=w^{3}$. And $\zeta=w^{3}$ maps the whole $\zeta$-plane onto the three sectors in the $w$-plane bounded by $w=r e^{i \theta}, \theta= \pm \pi / 3, \pi$. Hence the image of the whole $z$-plane by $z=\tanh w^{3}$ in the $w$-plane is as shown in Fig. 3.

Except the uninteresting case $f(z)=$ constant, the integrand $f(z)$ of the original integral has at least one singular point somewhere on the $z$ plane including $z=\infty$. One such point will be mapped by $z=\phi(w)=\tanh w^{m}$ onto an infinite array of singularities of $g(w)$ located in the finite $w$-plane on account of the multivaluedness of the inverse function

$$
w=\phi^{-1}(z)=(\operatorname{artanh} z)^{1 / m}
$$




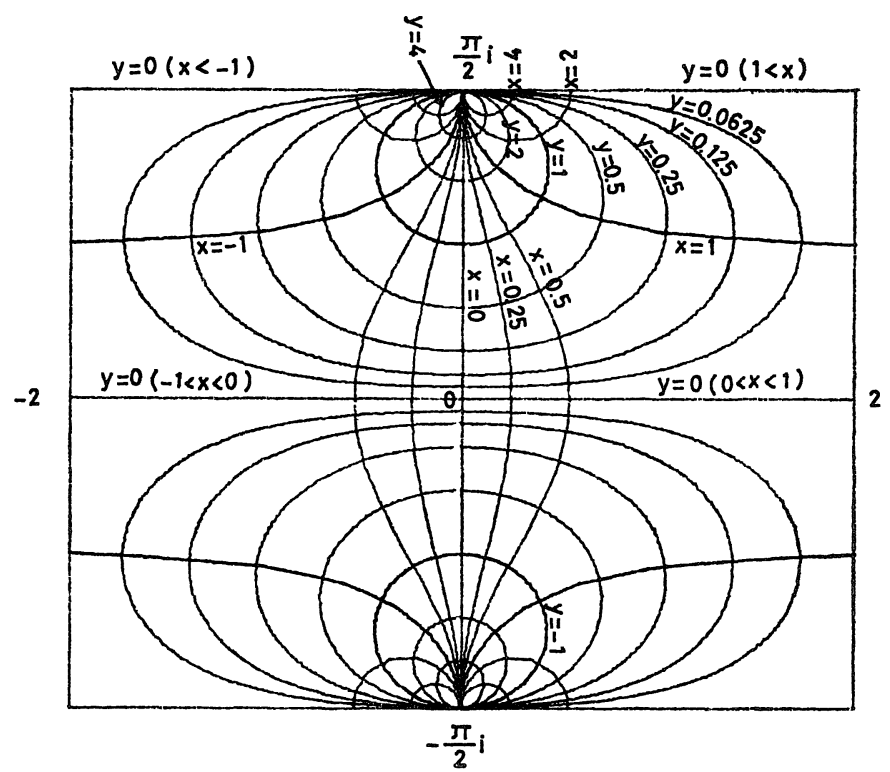

Fig. 2 Mapped images of the lines parallel to the real axis and the imaginary axis in the $z$-plane $(z=x+i y)$ by $w=$ $\operatorname{artanh} z$

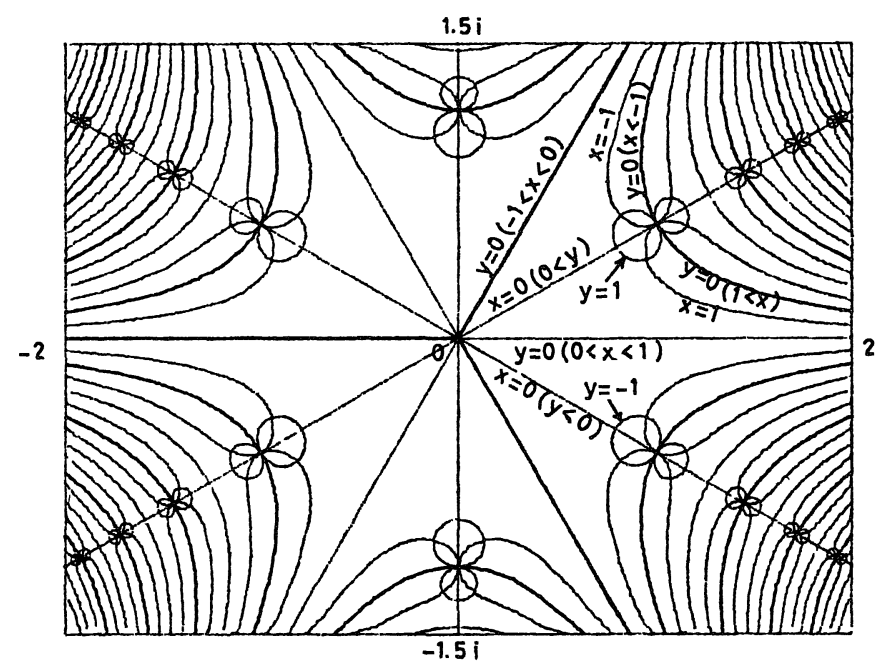

Fig. 3 Mapped images of the real and the imaginary axes and of the lines $x= \pm 1$ and $y= \pm 1$ in the $z$-plane $(z=x+i y)$ by $w=(\operatorname{artanh} z)^{1 / 3}$ 


$$
=\exp \left\{\frac{1}{m} \log \left(\frac{1}{2} \log \frac{1+z}{1-z}\right)\right\} .
$$

Let $z_{0}$ be one of the singularities in the $z$-plane including $z=\infty$. Then the corresponding singularities in the $w$-plane are given explicitly as

$$
\begin{aligned}
& w=\phi^{-1}\left(z_{0}\right)=\exp \left\{\frac{1}{m} \log \left(\frac{1}{2} \log \frac{1+z_{0}}{1-z_{0}}\right)\right\} \\
& =\left\{s^{2}+(t+k \pi)^{2}\right\}^{\frac{1}{2 m}} \exp \left\{\frac{i}{m}\left(\arctan \frac{t+k \pi}{s}+2 \pi l\right)\right\} ; \\
& \qquad k=0, \pm 1, \pm 2, \ldots ; l=0, \pm 1, \pm 2, \ldots \\
& \simeq(|k| \pi)^{\frac{1}{m}} \exp \left\{\left( \pm \frac{1}{2 m}+\frac{2 l}{m}\right) \pi i\right\}, \quad|k| \rightarrow \infty
\end{aligned}
$$

where

$$
\left\{\begin{array}{l}
s=\frac{1}{2} \log \left|\frac{1+z_{0}}{1-z_{0}}\right| \\
t=\frac{1}{2} \arg \frac{1+z_{0}}{1-z_{0}} .
\end{array}\right.
$$

That is, they are all distributed on $2 m$ infinite radial straight lines in the $w$-plane emanating from $w=0$ and making equal angles, as also seen from Fig. 3. Besides these singularities arising from those of $f(z)$, the integrand $f\left(\tanh w^{m}\right) m w^{m-1} \cosh ^{-2} w^{m}$ has an infinite number of poles which are due to the zeros of $\cosh w$.

Let $w_{0}$ be one of these singular points that are located nearest to the real axis in the $w$-plane. The imaginary part of $w_{0}$ is given from (2.a.7) if we put $k=l=0$

$$
\operatorname{Im} w_{0}=\sigma^{\frac{1}{2 m}} \sin \frac{\tau_{1}}{m},
$$

where

$$
\left\{\begin{array}{l}
\sigma=s^{2}+t^{2} \\
\tau_{1}=\arctan \frac{t}{s}=\arcsin \frac{t}{\sqrt{s^{2}+t^{2}}} .
\end{array}\right.
$$


Hence from (1.10) the order of magnitude of the error of (2.a.3) is given by

$$
\begin{aligned}
\left|\Delta I_{h}\right| & \simeq \exp \left(-\frac{2 \pi}{h} \sigma^{\frac{1}{2 m}}\left|\sin \frac{\tau_{1}}{m}\right|\right) \\
& \simeq \exp \left(-\frac{2 \pi}{m h}\left|\tau_{1}\right|\right), \quad m \rightarrow \infty .
\end{aligned}
$$

Note that $\sigma$ is of the order of unity or less except when $z_{0}$ is extremely close to \pm 1 and that $\left|\tau_{1}\right|<\pi / 2$.

Now, to get a rough estimate of the number $N$ of the sampling points necessary in order to keep the error $\left|\Delta I_{h}\right|$ below a given bound $\delta$, we put

$$
\left|\Delta I_{t}\right| \simeq\left|\Delta I_{h}\right| \simeq \delta
$$

where $\Delta I_{t}$ is the error due to the truncation of the summation (2.a.3). Then we have

$$
\exp \left(-\frac{2 \pi}{m h}\left|\tau_{1}\right|\right) \simeq \exp \left(-N^{m} h^{m}\right)
$$

and this equation yields

$$
h \simeq\left(\frac{2 \pi\left|\tau_{1}\right|}{m}\right)^{\frac{1}{m+1}} N^{-\frac{1}{m+1}} \simeq N^{-\frac{m}{m+1}}, \quad m \rightarrow \infty .
$$

Substituting this into (2.a.15) we have an error formula in terms of $N$ :

$$
\begin{aligned}
\left|\Delta I_{N}\right| & =\delta \simeq \exp \left\{-2 \pi\left(\sin \frac{\left|\tau_{1}\right|}{m}\right) N^{\frac{m}{m+1}}\right\} \\
& \simeq \exp \left(-\frac{2 \pi\left|\tau_{1}\right|}{m} N^{\frac{m}{m+1}}\right), \quad m \rightarrow \infty .
\end{aligned}
$$

If $m$ is varied for fixed $N$, the error $\left|\Delta I_{N}\right|$ decreases with increasing $m$, but from $\partial\left|\Delta I_{N}\right| / \partial m=0$ we see that it attains a minimum

$$
\left|\Delta I_{N}\right|_{\min } \simeq \exp \left(-\frac{2 \pi\left|\tau_{1}\right|}{\log N_{\min }} N_{\min }^{\frac{m}{m+1}}\right)
$$

at 


$$
m=\log N_{\min } \quad\left(N_{\min }=e^{m}\right),
$$

and then it turns to grow as $m$ is further increased. This situation is illustrated in Fig. 4.

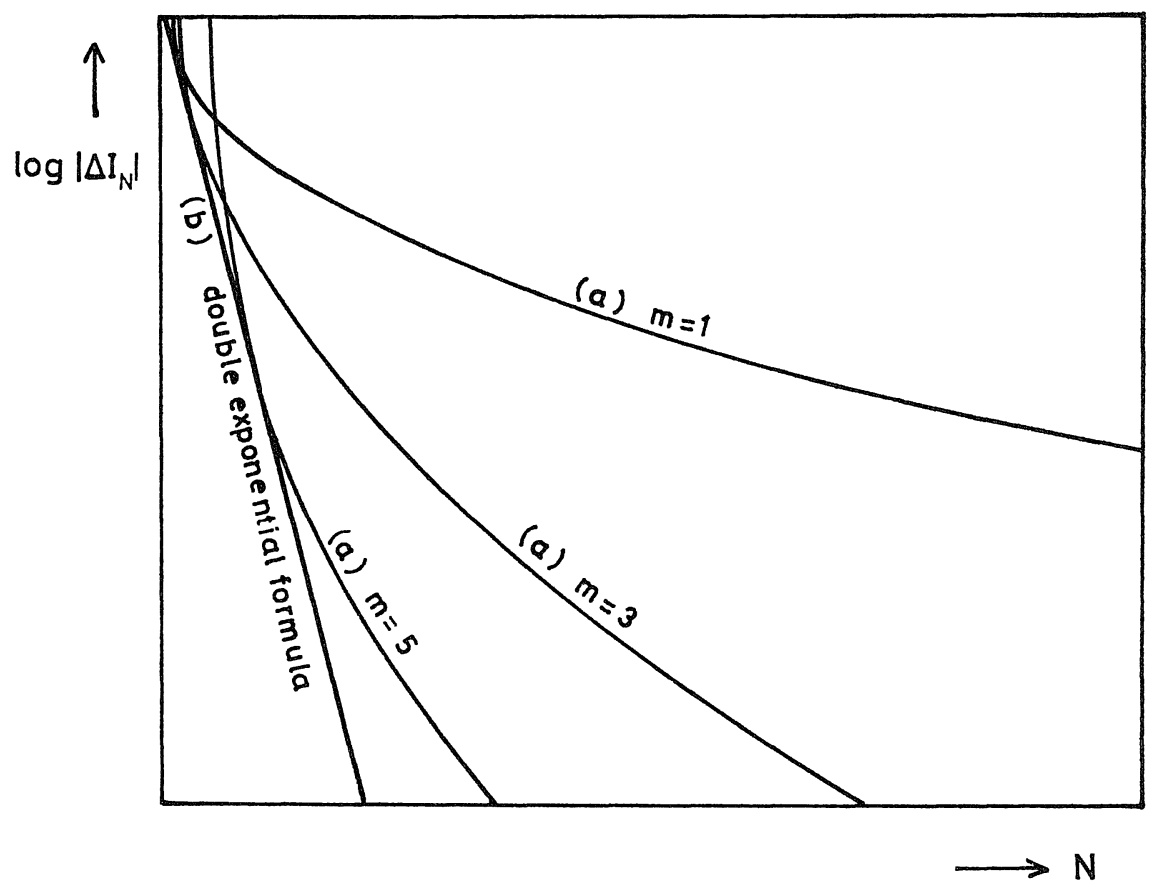

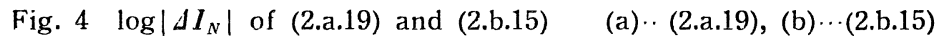

(2.b.1) $\quad x=\tanh \left(\frac{\pi}{2} \sinh u\right)$

- for which $|g(u)| \simeq \exp \left(-\frac{\pi}{2} \exp |u|\right)$ as $u \rightarrow \pm \infty$

As we have already seen, the error of (2.a.3) is essentially contributed by the specific singular point

$$
w_{0}=\left(s^{2}+t^{2}\right)^{\frac{1}{2 m}} \exp \left(\frac{i}{m} \arctan \frac{t}{s}\right)
$$

which correspond to $k=l=0$, while other singular points on the same line are not responsible for it. This situation can also be seen from Fig. 3. From the general theory of optimization, it will be intuitively clear that a better mapping function would be those for which two or more singular 
points make equal contribution to the error. Specifically, a better situation would be that in which the singular point $z_{0}$ in the $z$-plane is mapped onto any straight line in the $w$-plane which is parallel to the real axis. A mapping function satisfying this condition really exists and is given by a function of double exponential type, such as

$$
z=\tanh \left(\frac{\pi}{2} \sinh w\right)
$$

If we use (2.b.3) as a mapping function, we obtain the formula

$$
I_{h}=\frac{\pi}{2} h \sum_{n=-\infty}^{\infty} f\left(\tanh \left(\frac{\pi}{2} \sinh n h\right)\right) \frac{\cosh n h}{\cosh ^{2}\left(\frac{\pi}{2} \sinh n h\right)} .
$$

The mapping from the $z$-plane onto the $w$-plane is most easily understood by considering the intermediate plane $\zeta$ :

$$
\zeta=\frac{\pi}{2} \sinh w=\operatorname{artanh} z
$$

We already know that any singular point in the $z$-plane is mapped by $\zeta=$ $\operatorname{artanh} z$ onto an infinite array of points on a straight line which is parallel to the imaginary axis in the $\zeta$-plane. And then these points are mapped by $w=\operatorname{arsinh} \frac{\pi}{2} \zeta$ onto an infinite array of points in the $w$-plane lying asymptotically along lines $\operatorname{Im} w=(l \pm 1 / 2) \pi ; l=0, \pm 1, \pm 2, \ldots$ as we can easily understand from Fig. 5 in which the image of the whole $z$-plane with the lines $\operatorname{Re} z= \pm 1$ and $\operatorname{Im} z= \pm 1$ by $w=\operatorname{arsinh}\left(\frac{2}{\pi} \operatorname{artanh} z\right)$ is shown. This situation is also seen from the explicit representation of location of the singular points

$$
\text { (2.b.6) } \begin{aligned}
w= & l \pi i+(-1)^{l}\left[\operatorname { a r c o s h } \frac { 1 } { \pi } \left\{\sqrt{s^{2}+\left(t+\frac{\pi}{2}+k \pi\right)^{2}}\right.\right. \\
& \left.+\sqrt{s^{2}+\left(t-\frac{\pi}{2}+k \pi\right)^{2}}\right\} \pm i \arcsin \frac{1}{\pi}\left\{\sqrt{s^{2}+\left(t+\frac{\pi}{2}+k \pi\right)^{2}}\right. \\
& \left.\left.-\sqrt{s^{2}+\left(t-\frac{\pi}{2}+k \pi\right)^{2}}\right\}\right] ; k=0, \pm 1, \pm 2, \ldots ; l=0, \pm 1, \pm 2, \ldots \\
\simeq & l \pi i+(-1)^{l}\left(\operatorname{arcosh} 2 k \pm \frac{\pi}{2} i\right), \quad k \rightarrow \infty,
\end{aligned}
$$




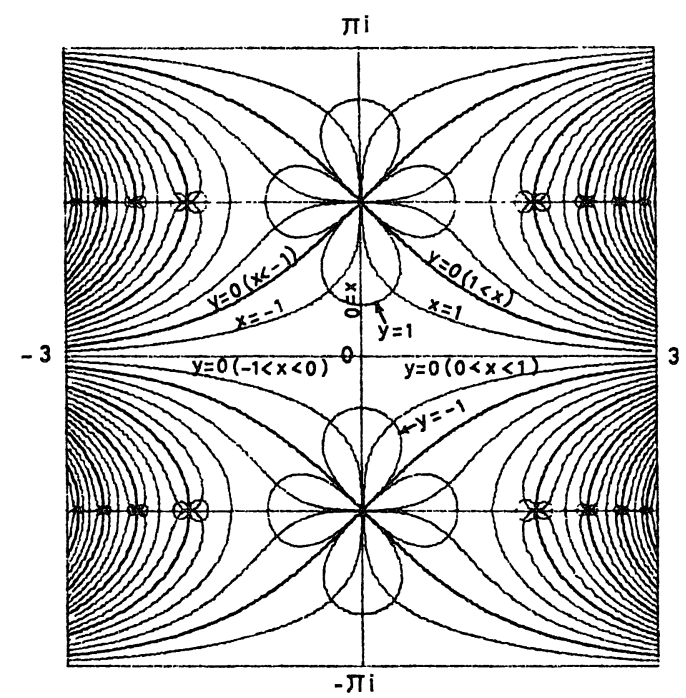

Fig. 5 Mapped images of the real and the imaginary axes and of the lines $x= \pm 1$ and $y= \pm 1$ in the $z$-plane $(z=x+i y)$ by $w=\operatorname{arsinh}\left(\frac{2}{\pi} \operatorname{artanh} z\right)$

where $s$ and $t$ are defined in (2.a.9) and (2.a.10).

Now let us see what occurs if the decay rate of $g(u)$ is further increased. For this purpose consider

$$
z=\tanh \left(\frac{\pi}{2} \sinh w^{m}\right), \quad m \geqq 3
$$

as an example. Then for $l=0$ and for large $k$ in (2.b.6) we have

$$
w=\left(\operatorname{arcosh} 2 k \pm \frac{\pi}{2} i\right)^{\frac{1}{m}}
$$

The line on which the singular points lie in the $\zeta$-plane $(\zeta=\operatorname{artanh} z)$ is now mapped by $\zeta=(\pi / 2) \sinh w^{m}$ onto a curve which approaches the real axis asymptotically as $|\operatorname{Re} w| \rightarrow \infty$ as seen from (2.b.8). Hence the path of integration must approach the real axis at both ends, and the main contribution to the error comes from this part of the contour and will give rise to a greater error, as will be evident in the example below. Hence we can say with much confidence that the decay rate of

$$
|g(u)| \simeq \exp \left(-\frac{\pi}{2} \exp |u|\right)
$$


corresponding to (2.b.3) is optimal for the quadrature efficiency.

It would be instructive to check the optimal property of (2.b.4) by explicit error estimation. The error of (2.b.4) is essentially determined by the singular points $w_{0}$ of (2.b.6) located nearest to the real axis. If we put $k=l=0$ we have

$$
\left|\Delta I_{h}\right| \simeq \exp \left(-\frac{2 \pi}{h}\left|\tau_{2}\right|\right)
$$

where

$$
\tau_{2}=\arcsin \frac{2 t}{\sqrt{s^{2}+\left(t+\frac{\pi}{2}\right)^{2}}+\sqrt{s^{2}+\left(t-\frac{\pi}{2}\right)^{2}}}
$$

from (2.b.6). If we truncate (2.b.4) at $\pm u_{\infty}= \pm N h$, the resulting truncation error is about

$$
\left|\Delta I_{t}\right| \simeq \exp \left(-\frac{\pi}{2} \exp u_{\infty}\right)
$$

and putting $\left|\Delta I_{t}\right|=\left|\Delta I_{h}\right|$ we have

$$
h=\frac{1}{N} \log \frac{4\left|\tau_{2}\right|}{h} .
$$

An approximate solution of the transcendental equation (2.b.13) will be obtained by using $h=1 / N$ as a zeroth approximation and substituting it into the right hand side of (2.b.13), yielding

$$
h \simeq \frac{1}{N} \log \left(4\left|\tau_{2}\right| N\right) .
$$

Thus we have an error estimate of (2.b.4) in terms of $N$ :

$$
\left|\Delta I_{N}\right| \simeq \exp \left\{-\frac{2 \pi\left|\tau_{2}\right|}{\log \left(4\left|\tau_{2}\right| N\right)} N\right\}
$$

We have now come to the step to compare the result (2.b.15) with (2.a.19). Note that $\left|\tau_{1}\right|$ and $\left|\tau_{2}\right|$ are quantities with the same order of magnitude as seen from (2.a.13) and (2.b.11). As is evident on comparing the two results, (2.b.15) is the approximate lower limit of (2.a.19). 
In other words, if we plot $\log \left|\Delta I_{N}\right|$ as a function of $N$, we will find that (2.b.15) gives an approximate envelope of the family of curves given by (2.a.19) somewhat as shown in Fig. 4, since $\left|\Delta I_{N}\right|_{\mathrm{min}}$ in (2.a.21) is approximately equal to (2.b.15). Therefore we can suppose with all probability that a mapping function of the form

$$
x=\phi(u)=\tanh \left(\frac{\pi}{2} \sinh u\right)
$$

is optimal with respect to the efficiency.

The point that has been made in this section can be summarized to the statement that the transformed function $g(u)$ should have a decay property

$$
g(u) \simeq \exp \left(-\frac{\pi}{2} \exp |u|\right), \quad u \rightarrow \pm \infty
$$

or, equivalently, that $g(w)$ should have an infinite array of singular points lying asymptotically parallel to the real axis.

\section{§3. Double Exponential Formulas}

In the preceding section we have found the best quadrature formula for integrals over $(-1,1)$ among those obtained by the variable transformation, and we have seen that what is essential in the transformation is the decay property at $u \rightarrow \pm \infty$ of the transformed function $g(u)$ which is to be integrated over $(-\infty, \infty)$. That is, it is best to choose a mapping function that results in such a transformed integrand $g(u)$ as behaves like (2.b.17). And hence not only for integrals of $f(x)$ over $(-1,1)$ but also for those over any interval including $(0, \infty)$ or $(-\infty, \infty)$ we would obtain the best quadrature formula by investigating the analytic behavior of $f(x)$ and by choosing a mapping function according to the principle that $g(u)$ should have the decay property as (2.b.17), provided that $f(x)$ is analytic and integrable over the given interval. For instance, if the given integral has by itself the property (2.b.17), as in $\int_{-\infty}^{\infty} \exp (-\cosh x+\operatorname{iax}) d x$, for example, no variable transformation is needed, the direct application of the trapezoidal rule with an equal mesh size giving the best result.

We will give here a few typical examples of the formulas. 
(3-a) Finite interval $(-1,1)$

$$
\begin{gathered}
I=\int_{-1}^{1} f(x) d x \\
x=\tanh \left(\frac{\pi}{2} \sinh u\right) \\
I_{h}=\frac{\pi}{2} h \sum_{n=-\infty}^{\infty} f\left(\tanh \left(\frac{\pi}{2} \sinh n h\right)\right) \frac{\cosh n h}{\cosh ^{2}\left(\frac{\pi}{2} \sinh n h\right)} .
\end{gathered}
$$

(3-b) Half-infinite interval $(0, \infty)$

$$
\begin{gathered}
I=\int_{0}^{\infty} f(x) d x \\
x=\exp \left(\frac{\pi}{2} \sinh u\right) \\
I_{h}=\frac{\pi}{2} h \sum_{n=-\infty}^{\infty} f\left(\exp \left(\frac{\pi}{2} \sinh n h\right)\right)(\cosh n h) \exp \left(\frac{\pi}{2} \sinh n h\right) .
\end{gathered}
$$

Many of integrals over $(0, \infty)$ encountered in the practical problems have an exponential factor in their integrands:

$$
f(x)=f_{1}(x) e^{-x}
$$

In this case the integrand has already the decay property as a single exponential function at $u \rightarrow+\infty$, and hence we have only to choose such a mapping function as adds one more exponential decay at $u \rightarrow+\infty$, together with the double exponential decay at $u \rightarrow-\infty$.

$$
\begin{gathered}
x=\exp (u-\exp (-u)) \\
I_{h}=h \sum_{n=-\infty}^{\infty} f(\exp (n h-\exp (-n h)))\{1+\exp (-n h)\} \cdot \\
\cdot \exp (n h-\exp (-n h)) .
\end{gathered}
$$

(3-c) Infinite interval $(-\infty, \infty)$

$$
I=\int_{-\infty}^{\infty} f(x) d x
$$




$$
\begin{gathered}
x=\sinh \left(\frac{\pi}{2} \sinh u\right) \\
I_{h}=\frac{\pi}{2} h \sum_{n=-\infty}^{\infty} f\left(\sinh \left(\frac{\pi}{2} \sinh n h\right)\right)(\cosh n h) \cosh \left(\frac{\pi}{2} \sinh n h\right)
\end{gathered}
$$

This formula is used in order to change a slowly convergent integral into a rapidly convergent one.

Since each of these mapping functions gives $|g(u)|$ which decays as a double exponential function of $u$ as $u \rightarrow \pm \infty$, as seen in (2.b.17), we will call any quadrature formula having such a property as double exponential formula. We can find many other double exponential formulas for different types of integrals.

The double exponential formulas shown above have a practical advantage that the positions of the sampling points and the weights can easily be generated in the process of integration in the computer with the aid of the exponential function. Since the distribution of the sampling points is equidistant, one can make use of the values computed at preceding steps when one employs an automatic procedure in which the step size is halved repeatedly until the required precision is attained.

In the actual computation of improper integrals one should be aware of the loss of significant figures due to cancellation occurring as $x$ tends to the end points of the interval. For example, it occurs as $x \rightarrow \pm 1$ when the integrand in $(3.1)$ has a factor $(1+x)^{\alpha}(1-x)^{\beta}$. In such a case one can avoid the loss of significant figures by a direct substitution of

$$
\begin{aligned}
t=1 \pm x & =\frac{\exp \left( \pm \frac{\pi}{2} \sinh u\right)}{\cosh \left(\frac{\pi}{2} \sinh u\right)} \\
& \simeq 2 \exp ( \pm \pi \sinh u), \quad x \rightarrow \mp 1 .
\end{aligned}
$$

\section{§4. Numerical Examples}

The double exponential formulas are applied to the following examples and are compared with formulas obtained by other mapping functions.

$$
\int_{-1}^{1} \frac{d x}{(x-2)(1-x)^{1 / 4}(1+x)^{3 / 4}}=-1.9490 \ldots
$$


(4-ii)

$$
\int_{-1}^{1} \frac{\cos \pi x}{\sqrt{1-x}} d x=-0.69049 \ldots
$$

(4-iii)

$$
\int_{0}^{\infty} \frac{\exp (-1-x)}{1+x} d x=E_{1}(1)=0.21938 \ldots
$$

(4-iv)

$$
\int_{-\infty}^{\infty} \frac{d x}{\left(1+x^{2}\right)^{5 / 4}}=2.3962 \ldots
$$

$(4-v)$

$$
\int_{-\infty}^{\infty} \frac{d x}{1+x^{4}}=2.2214 \ldots
$$

The transformations used for the sake of comparison are as given below for each interval concerned:

Interval $(-1,1)$

(4-a) TANH-rule

$$
x=\tanh u \quad(|g(u)| \simeq \exp (-|u|) \text { as } u \rightarrow \pm \infty)
$$

(4-b) ERF-rule

$$
\begin{aligned}
x=\operatorname{erf} u=\frac{2}{\sqrt{\pi}} \int_{0}^{u} \exp \left(-t^{2}\right) d t \\
\quad\left(|g(u)| \simeq \exp \left(-u^{2}\right) \text { as } u \rightarrow \pm \infty\right)
\end{aligned}
$$

(4-c) TSH3-rule

$$
x=\tanh \left(\frac{\pi}{2} \sinh u^{3}\right)
$$

(Given as an example of a mapping function which gives a decay rate greater than double exponential)

(4-d) IMT-rule [4]

$$
x=\frac{1}{Q} \int_{-1}^{u} \exp \left(-\frac{2}{1+t}-\frac{2}{1-t}\right) d t,
$$

$$
Q=\int_{-1}^{1} \exp \left(-\frac{2}{1+t}-\frac{2}{1-t}\right) d t
$$

(Mapping the interval $(-1,1)$ onto itself) 
Interval $(0, \infty)$ (with such an integrand as (3.7))

(4-e) EXP-rule

$$
\begin{aligned}
& x=\exp u \\
& (|g(u)| \simeq \exp (-\exp |u|) \text { as } u \rightarrow+\infty,|g(u)| \simeq \exp (-|u|) \\
& \text { as } u \rightarrow-\infty)
\end{aligned}
$$

(4-f) LERF-rule

$$
x=\log \frac{2}{1-\operatorname{erf} u}
$$

(Giving exactly the same weight $\exp \left(-n^{2} h^{2}\right)$ as in the case of the $\mathrm{ERF}$-rule (4.2), so that $|g(u)| \simeq \exp \left(-u^{2}\right)$ as $\left.u \rightarrow \pm \infty\right)$

Interval $(-\infty, \infty)$

(4-g) SINH-rule

$$
x=\sinh u \quad(|g(u)| \simeq \exp (-|u|) \text { as } u \rightarrow \pm \infty)
$$

(4-h) TAN-rule

$$
\begin{gathered}
x=\tan u \\
I_{h}=h \sum_{n=-N / 2}^{N / 2-1} f(\tan n h) \frac{1}{\cos ^{2} n h}, \quad h=\frac{\pi}{N} \quad(N=\text { even })
\end{gathered}
$$

(Mapping the infinite interval $(-\infty, \infty)$ into a finite interval $(-\pi / 2, \pi / 2)$, and applicable only to functions regular at $x= \pm \infty)$

Figs. 6-10 show the errors of various formulas as function of the number $N$ of sampling points actually required ${ }^{1)}$.

In the case of finite interval, the transformation $x=\tanh \left(\frac{\pi}{2} \sinh u^{3}\right)$ is inferior to the double exponential one, as has been anticipated. The IMT-rule is not good either, as discussed in $\$ 1$. It should be noted that,

1) In obtaining these plots a special care has been taken to adjust the step size and the truncation point so as to attain the highest precision for each $N$. Although this was necessary for the purpose of obtaining a smooth plot, it is not at all necessary in practical application of our formulas. 
as is shown in Fig. 8, the double exponential formula (3.9) is even better than the Laguerre-Gauss formula for the specific example (4-iii) which has an exponential factor $e^{-x}$ in the integrand, and for which it is commonly agreed as reasonable to apply the Laguerre-Gauss formula. In the example $(4-\mathrm{v})$ in which the integrand is regular at infinity, the TAN-rule is seen to be better than the double exponential formula. This is not hard to understand since the double exponential formulas are "robust" formulas which are rather insensitive to singularities of various types that may occur at the end points.

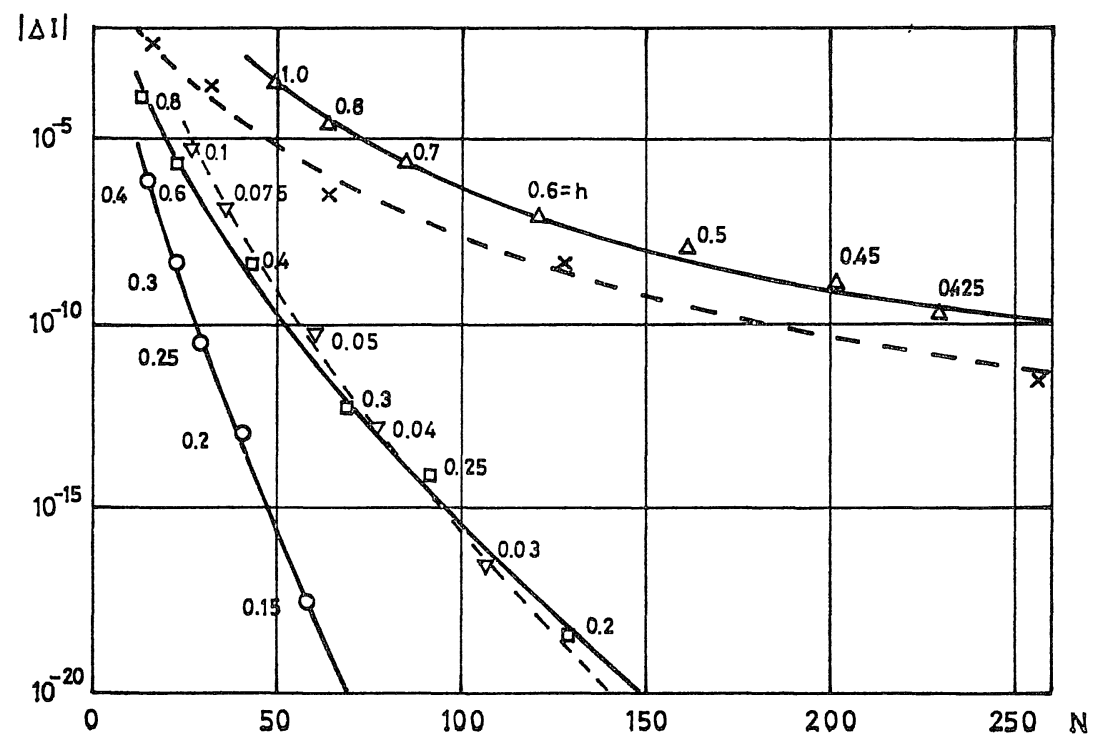

Fig. 6 The computed error of $\int_{-1}^{1} 1 /\left\{(x-2)(1-x)^{1 / 4}(1+x)^{3 / 4}\right\} d x$

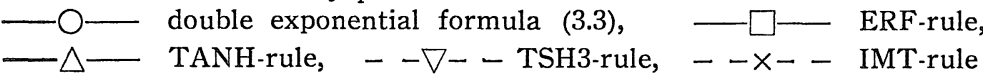




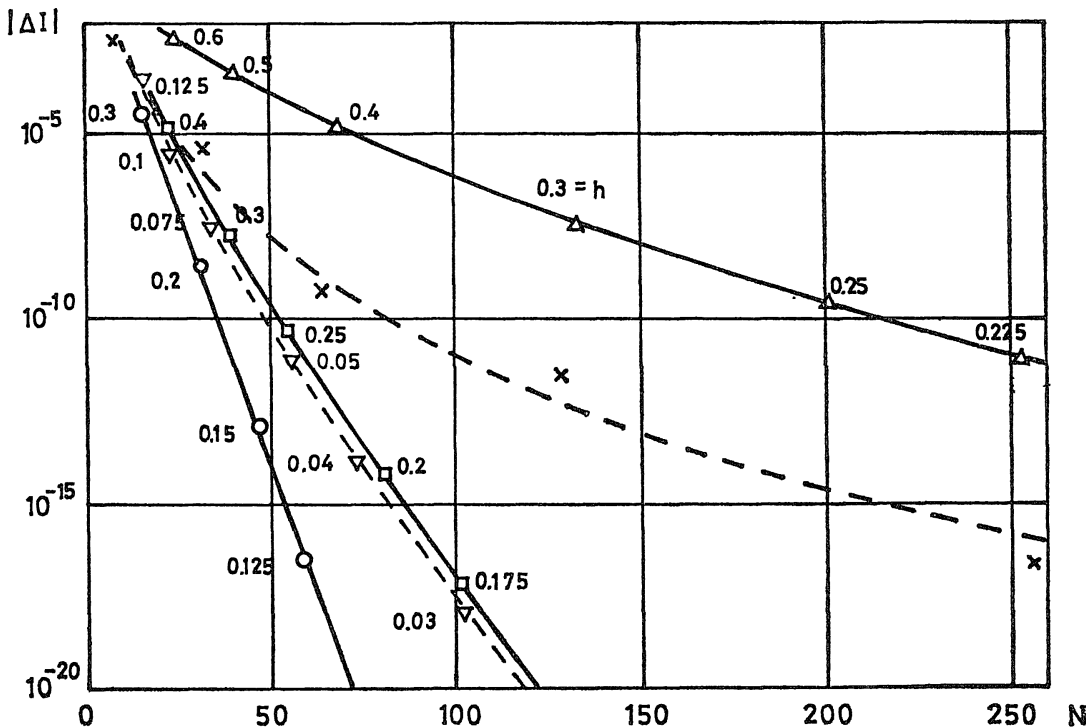

Fig. 7 The computed error of $\int_{-1}^{1}(\cos \pi x) / \sqrt{1-x} d x$

$-\mathrm{O}-$ double exponential formula (3.3), $\quad-\square-\square-$ ERF-rule,

$-\triangle-$ TANH-rule, $--\nabla--$ TSH3-rule, $--x--$ IMT-rule

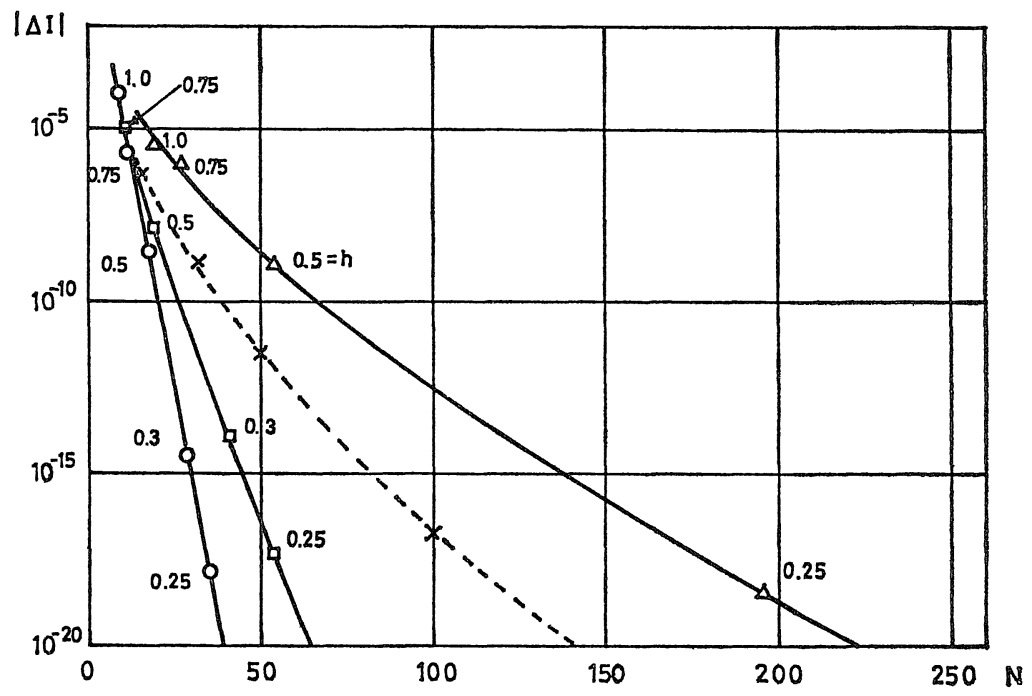

Fig. 8 The computed error of $\int_{0}^{\infty} \exp (-1-x) /(1+x) d x$

$-\mathrm{O}-$ double exponential formula (3.9), - $\square-$ LERF-rule,

$-\triangle-$ EXP-rule, $\quad--x--$ Laguerre-Gauss formula 


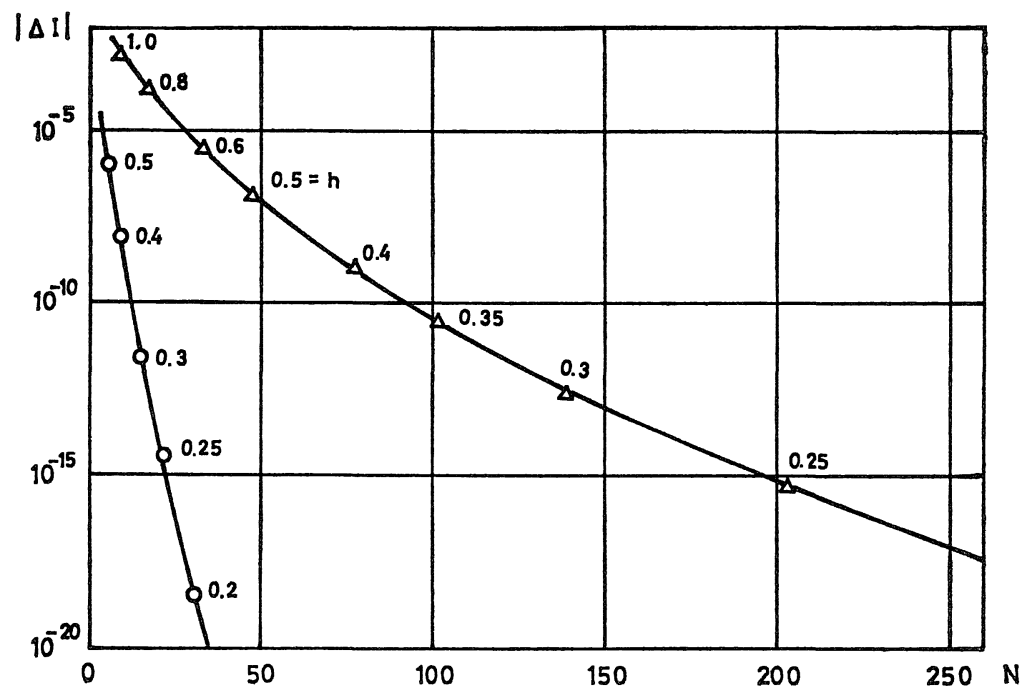

Fig. 9 The computed error of $\int_{-\infty}^{\infty}\left(1+x^{2}\right)^{-5 / 4} d x$ $-\mathrm{O}-$ double exponential formula (3.12), $\longrightarrow \triangle \longrightarrow$ SINH-rule

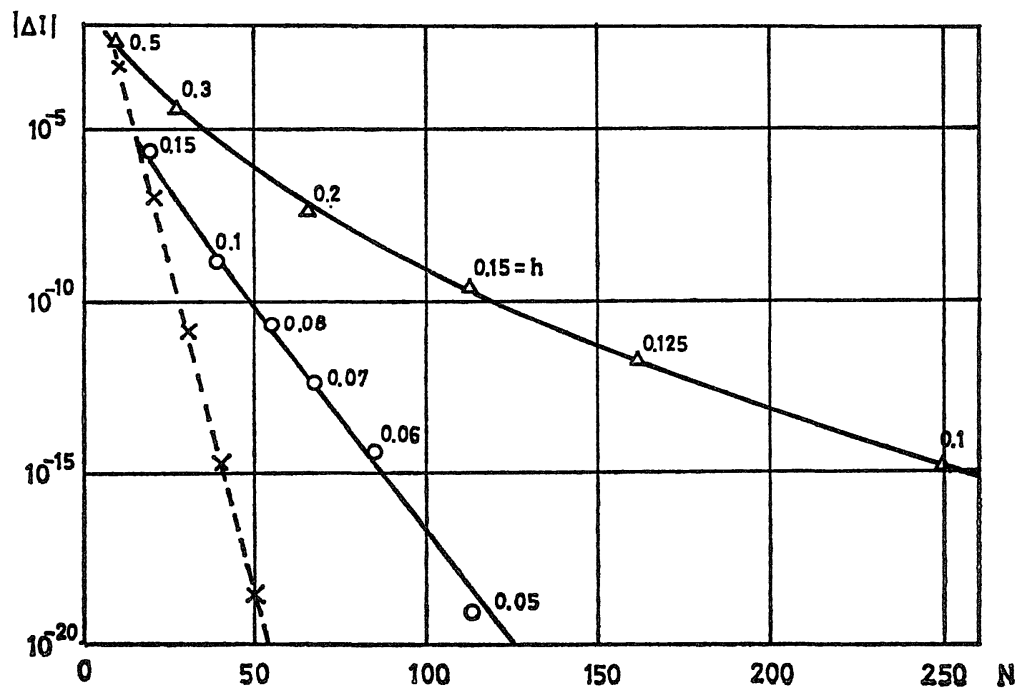

Fig. 10 The computed error of $\int_{-\infty}^{\infty}\left(1+x^{4}\right)^{-1} d x$

$-\mathrm{O}-$ double exponential formula (3.12), $\quad-\triangle-$ SINH-rule, $--x--$ TAN-rule 


\section{Summary}

It has been shown by analytic means that, among the various variable transformations that can be applied to convert a given definite integral into rapidly convergent integrals over $(-\infty, \infty)$, those which give a double exponential decay of the resulting integrands at $\pm \infty$ are in general the most efficient ones for the purpose of numerical quadrature using trapezoidal rule with a uniform sampling interval. This statement has been confirmed numerically by applying several formulas having that property to a few simple quadrature problems.

Note added in proof: In our formulas, owing to the exponential asymptotic dependence of the error on the inverse step size (cf. eq. (2.b.10)), halving the step size approximately doubles the number of significant figures. This fact makes them especially suitable for very high precision calculation and for automatic integration, and it has been shown on several test problems that our formula for finite interval compares favorably to the Romberg method even for regular integrands, whenever a precision of about fifteen decimal places or more are needed.

\section{References}

[1] Takahasi, H. and Mori, M., Error Estimation in the Numerical Integration of Analytic Functions, Report of the Computer Centre, Univ. of Tokyo, 3 (1970), 41108.

[2] Schwartz, C., Numerical Integration of Analytic Functions, J. Computer Physics, 4 (1969), 19-29.

[3] Takahasi, H. and Mori, M., Quadrature Formulas Obtained by Variable Transformation, Numer. Math., 21 (1973), 206-219.

[4] Iri, M., Moriguti, S. and Takasawa, Y., On a Certain Quadrature Formula (in Japanese), "Kokyuroku" of the Research Institute for Mathematical Sciences, Kyoto Univ., No. 91 (1970), 82-118. 
